\title{
A Cross-Mode Universal Digital Pre-Distortion Technology for Low-Sidelobe Active Antenna Arrays in 5G and Satellite Communications
}

\author{
Yunfeng Li ${ }^{1,2}{ }^{D}$, Yonghui Huang ${ }^{1, *}$, Martin Hedegaard Nielsen ${ }^{3}$, Feridoon Jalili ${ }^{3}$, Wei Wei ${ }^{3}$, , Jian Ren ${ }^{4}$, \\ Yingzeng Yin ${ }^{4}$, Ming Shen ${ }^{3}$ and Gert Frolund Pedersen ${ }^{3}$
}

check for updates

Citation: Li, Y.; Huang, Y.; Nielsen, M.H.; Jalili, F.; Wei, W.; Ren, J.; Yin, Y.; Shen, M.; Pedersen, G.F. A Cross-Mode Universal Digital Pre-Distortion Technology for Low-Sidelobe Active Antenna Arrays in 5G and Satellite Communications. Electronics 2021, 10, 2031. https:// doi.org/10.3390/electronics10162031

Academic Editors: Marco Pirola and Vittorio Camarchia

Received: 15 July 2021

Accepted: 17 August 2021

Published: 22 August 2021

Publisher's Note: MDPI stays neutral with regard to jurisdictional claims in published maps and institutional affiliations.

Copyright: (c) 2021 by the authors. Licensee MDPI, Basel, Switzerland. This article is an open access article distributed under the terms and conditions of the Creative Commons Attribution (CC BY) license (https:// creativecommons.org/licenses/by/ $4.0 /)$.
1 National Space Science Center, Chinese Academy of Sciences, Beijing 100190, China; yli@es.aau.dk or liyunfeng17@mails.ucas.ac.cn

2 School of Electronic, Electrical and Communication Engineering, University of Chinese Academy of Sciences, Beijing 100049, China

3 Department of Electronic Systems, Aalborg University, 9220 Aalborg, Denmark; mhni@es.aau.dk (M.H.N.); fja@es.aau.dk (F.J.); weiweisucceed@gmail.com (W.W.); mish@es.aau.dk (M.S.); gfp@es.aau.dk (G.F.P.)

4 National Key Laboratory of Antennas and Microwave Technology, Xidian University, Xi'an 710071, China; renjian@xidian.edu.cn (J.R.); yzyin@mail.xidian.edu.cn (Y.Y.)

* Correspondence: yonghui@nssc.ac.cn; Tel.: +86-186-1832-9725

\begin{abstract}
A cross-mode universal digital pre-distortion (CMUDPD) technology is proposed here to linearize low-sidelobe active antenna arrays with non-uniform fixed power levels for each branch, which are desired in satellite communications with stringent requirements to minimize interference. In low-sidelobe arrays formed by nonuniform amplitude excitation, conventional digital pre-distortion (DPD) techniques require multiple feedback paths for either one-to-one or average linearization of the PAs, which increases system complexity and is infeasible for large-scale arrays. This is because the power amplifiers (PAs) usually operate in different modes where the supply voltages, bias voltages, and input power levels are different. The proposed CMUDPD method aims at solving this issue by intentionally arranging the PAs to work in different modes but with shared nonlinear characteristics. Based on the nonlinear correlation established among the PAs' different operating modes, a single feedback path is sufficient to capture the common nonlinearity of all the PAs and determine the parameters of the CMUDPD module. The concept is explained in theory and validated by simulations and experiments using GaN PAs operating with three significantly different output power levels and two orthogonal frequency division multiplexing (OFDM) signal bandwidths.
\end{abstract}

Keywords: cross-mode universal digital pre-distortion (CMUDPD); low-sidelobe active antenna arrays; power amplifiers (PAs); nonlinear correlation

\section{Introduction}

Active antenna arrays are the most critical enablers in the development of highperformance $5 \mathrm{G}$ and satellite communication systems owing to their beam-steering capability of confining power emissions produced by radio-frequency (RF) power amplifiers (PAs) [1-3]. In order to improve the efficiency of PAs, the PAs are commonly driven to their saturation modes, which features high nonlinearities and may lead to severe distortion of the signals. Moreover, to meet the need to increase spectrum capacity and multiplexing, advanced modulation schemes, such as orthogonal frequency division multiplexing (OFDM) and similar derivatives, are being used for next-generation high throughput satellite communication systems [4]. These signals usually feature a high peak-to-average power ratio (PAPR) and are sensitive to the inherent nonlinearity distortions caused by RF PAs [1].

Several technologies for the linearization of uniform arrays, which can be categorized into two groups. One group of conventional one-to-one array digital pre-distortion (DPD) techniques is illustrated in Figure 1a, where each PA has a separatedfeedback path. The 
number of feedback paths equals the number of PAs [5]. This is acceptable for small-scale arrays with a few PAs, but it would be extremely challenging for large-scale arrays that consist of hundreds or thousands of PAs [6]. The linearization of arrays consumes abundant amounts of operation power and makes the system unnecessarily complex, since both a analog-to-digital converter (ADC) and coupler are essential components for each feedback path $[7,8]$, as shown in Figure 1a. Additionally, nowadays, active arrays tend to be more integrated and compact [9], making one-to-one pre-distortion of PAs infeasible.

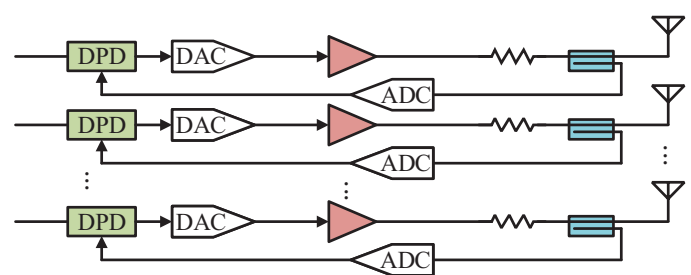

(a)

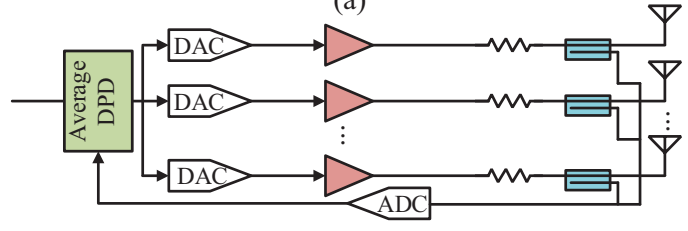

(b)

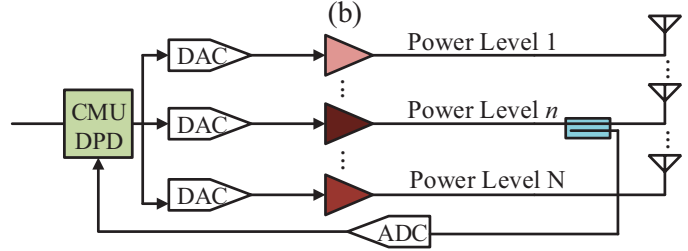

(c)

Figure 1. Block diagram of digital pre-distortion (DPD) techniques used for non-uniform amplitude excitation active antenna arrays: (a) one-to-one DPD [5], (b) average DPD [10], and (c) the proposed cross-mode universal digital pre-distortion (CMUDPD).

To reduce the feedback-path quantity, the average DPD system with one feedback path was proposed to linearize the active array, as shown in Figure 1b [10]. Only one ADC component remains in the average DPD system, which reduce sits ADC power consumption compared with Figure 1a. However, the linearization performance is highly dependent on the consistency of PAs, specifically the gain factor, which is used to synthesize the pre-distortion function. Moreover, a large amount of couplers is still mandatory. Various authors [11-16] attempted to model and pre-distort the non-uniform active arrays. However, only conditions with different input power levels (leading to various operation regimes) were considered in these works, while optimizations of supply voltages and bias voltages were not covered. This means that several PAs will not operate in their compression regions. Accordingly, these PAs operating in low power levels or even linear regions cannot reach their optimum efficiency. Therefore, the power efficiency achieved by [11-16] may be limited.

Additionally, for multi-antenna systems in satellite communications, the consistency of PAs in an active array cannot be guaranteed [17]. Power amplifiers tend to operate in different modes due to their imperfections (e.g., the manufacturing tolerance) and different local environmental conditions (e.g., the operating temperature). However, the sidelobe level is an essential specification of satellite antenna arrays, as it characterizes the capability of arrays to suppress the interference. In order to realize low sidelobes while maintaining high power efficiency, PAs can be intentionally arranged to operate in different saturation modes with desired power levels [18-22]. However, it is infeasible to directly apply the existing DPD methods designed for signal PA or uniform power antenna arrays to linearize low-sidelobe active antenna arrays with non uniform power distribution [23-28]. Therefore, an effective linearization technique for low-sidelobe active antenna arrays is needed. 
In this paper, a cross-mode universal digital pre-distortion (CMUDPD) technique is proposed to linearize low-sidelobe active antenna arrays, as depicted in Figure 1c. For the proposed CMUDPD, a single feedback path is utilized, which is sufficient to capture the nonlinear correlation of all PAs. Notably, PAs operate in different modes while at the same compression point, where the supply voltages, bias voltages, and input power levels are different.

In Table 1, the proposed CMUDPD is compared with several representative state-ofthe-art DPD methods. Several DPD technologies studied in $[5,10,12,24]$ consider uniform arrays. Although parametric variations in $P_{\text {in }}$ are involved in [10,12], their amplitudes are relatively small compared to those in low-sidelobe arrays. In [10], the resultant average DPD coefficients were obtained by training a set of random amplitude and phase data following the normal distribution. For the array DPD method studied in [20] for lowsidelobe arrays, it is mandatory to synthesize all PAs' outputs by two steps, i.e., linearizing the higher driven PAs, followed by cancelling compression by the lower driven PAs, to form a single feedback signal. Consequently, its linearization performance is highly dependent on the consistency of the PAs, and a large amount of couplers is required. Compared with [20], the proposed CMUDPD does not require the synthesis of all PAs' outputs, and an arbitrary PA's output can be used as the feedback signal. Moreover, different operation modes with variations in PA supply voltage $V_{\mathrm{DD}}$, bias voltage $V_{\mathrm{GG}}$, and input power $P_{\text {in }}$ can be fully linearized using the proposed CMUDPD. Compared to our conference publication [29], this paper presents the theoretical foundation explaining why the proposed CMUDPD can linearize different PA operation modes, as explained in Section 2. Moreover, experimental results are presented to show that the proposed CMUDPD can achieve an averaged adjacent channel power ratio (ACPR) suppression of $13 \mathrm{~dB}$.

Table 1. The comparison of the proposed solution with the state-of-the art methods. N: number of antenna elements, $P_{\mathrm{in}}$ : input power, $V_{\mathrm{DD}}$ : PA supply volage, $V_{\mathrm{GG}}$ : PA bias voltage.

\begin{tabular}{cccccc}
\hline Method & Array Types & $\begin{array}{c}\text { Feedback Path } \\
\text { Number }\end{array}$ & $\begin{array}{c}\text { DPD Unit } \\
\text { Number }\end{array}$ & $\begin{array}{c}\text { Synthesis of PAs } \\
\text { Outputs }\end{array}$ & $\begin{array}{c}\text { Variable Parameters } \\
\text { Considered }\end{array}$ \\
\hline One-to-one DPD [5] & Uniform & $\mathrm{N}$ & $\mathrm{N}$ & Not required & - \\
Average DPD [10] & Uniform & 1 & 1 & Required & $P_{\text {in }}$ \\
BO-DPD [12] & Uniform & $1<\mathrm{n}<\mathrm{N}$ & 1 & Required & $P_{\text {in }}$ \\
Adaptive DPD [24] & Uniform & 1 & 1 & Required & - \\
Array DPD [20] & Low-sidelobe & 1 & 1 & Required & $P_{\text {in }}$ \\
Proposed CMUDPD & Low-sidelobe & 1 & 1 & Not required & $V_{\mathrm{DD}}, V_{\mathrm{GG}}, P_{\text {in }}$ \\
\hline
\end{tabular}

This paper is organized into five sections. Section 2 introduces the principle of the proposed CMUDPD scheme. The simulation setup and results are illustrated in Section 3. Section 4 presents the experimental results and discussion. Finally, the conclusion of this work is proided in Section 5.

\section{Principle of the CMUDPD Technique}

Understanding the physical distortion generation mechanisms is critical to designing a linear low-sidelobe antenna array. In this section, the structure of CMUDPD for a lowsidelobe active antenna array is described in detail.

The methods for generating a low-sidelobe active array antenna include using attenuators cascaded following power amplifiers to obtain the desired power levels, or using a nonuniform amplitude feed network [30,31]. In the first case, attenuators are located at the output side of each PA. It is obvious that the attenuators may consume a large amount of power, and they also increase the risk of failure due to heat generation. The available space on a satellite is very limited, and it is highly desired to use fewer feedback paths to achieve the linearization of a low-sidelobe antenna array. The existing low-sidelobe array antenna adopts the aperture amplitude weighted design process [32-35]. The unequal microstrip amplitude feed network is designed to achieve the low-sidelobe characteristics of an array. 
The method aims to drive the PAs with different input power levels. From the energy point of view, this situation, at least, does not waste a large amount of power. Therefore, a structure using a small signal unequal power divider is considered in the subsequent analysis, as shown in Figure 2.

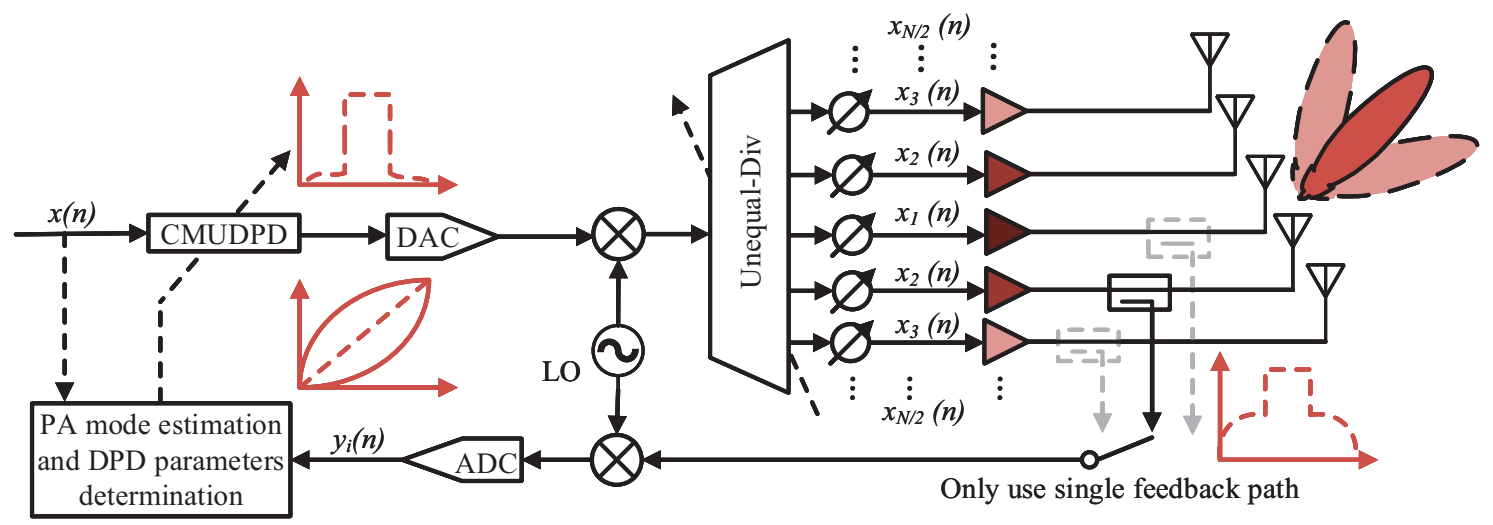

Figure 2. The system architecture of non-uniform amplitude excitation arrays with the proposed CMUDPD linearization technique.

The input-output relationship of the power amplifier is mainly reflected in the inherent static nonlinear characteristics and memory effects [36,37]. In Figure $3 a, \mathrm{PA}_{i}$ and $\mathrm{PA}_{j}$ represent two PAs in a phased array operating at the $1 \mathrm{~dB}$ compression point under mode $i$ and mode $j$, respectively. The actual input-output relationship of $\mathrm{PA}_{i}$ can be expressed as

$$
y_{i}(t)=G_{i} \cdot g_{i}\left(x_{i}(t)\right),
$$

where $G_{i}$ is a constant complex number and indicates expected gain values of $\mathrm{PA}_{i}$. It should be noted that $g_{i}(\cdot)$ represents the shape function of the gain curve, and $g_{i}^{-1}(\cdot)$ is the inversion of the gain curve $g_{i}(\cdot)$. Then, the ideal pre-distortion signal to $\mathrm{PA}_{i}$, i.e., $x_{i}$ DPD $(t)$, can be expressed as

$$
x_{i \_ \text {DPD }}(t)=G_{i} \cdot g_{i}^{-1}\left(x_{i}(t)\right) .
$$

So, the output signal after the pre-distortion, i.e., $y_{i \_D P D}$, can be obtained as follows:

$$
y_{i \_\mathrm{DPD}}(t)=G_{i} \cdot g_{i}\left(g_{i}^{-1}\left(x_{i}(t)\right)\right)=G_{i} \cdot x_{i}(t),
$$

which indicates that $\mathrm{PA}_{i}$ is linearized.

The simulation and experiment results show that $\mathrm{PA}_{i}$ and $\mathrm{PA}_{j}$ can have similar characteristics by properly tuning their supply and bias voltages. The similar characteristics mean that the gain vs. input power curve of $\mathrm{PA}_{i}$ can be replicated by moving that of $\mathrm{PA}_{j}$ vertically and horizontally, as shown in Figure $3 \mathrm{~b}$. In other words, the gain vs. input power curves of $\mathrm{PA}_{i}$ and $\mathrm{PA}_{j}$ have a similar shape, and the gains of $\mathrm{PA}_{i}$ and $\mathrm{PA}_{j}$ (i.e., $G_{i}$ and $G_{j}$ ) feature the following relationship:

$$
G_{j}=G_{i} \cdot 10^{\alpha / 20},
$$

where $\alpha$ is a vertical constant as indicated in Figure 3b. In this situation, the actual output of $\mathrm{PA}_{j}$ can be written as

$$
y_{j}(t) \approx G_{i} \cdot g_{i}\left(x_{i}(t) \cdot 10^{-\beta / 20}\right) \cdot 10^{\alpha / 20},
$$


where $\beta$ is the horizontal constant as indicated in Figure $3 \mathrm{~b}$. The pre-distortion signal of $\mathrm{PA}_{j}$, $x_{j \_\mathrm{DPD}}(t)$ can be obtained by shifting the power level of $x_{i \_\mathrm{DPD}}(t)$ by $10^{\beta / 20}$, which provides

$$
x_{j \_\mathrm{DPD}}(t)=x_{i \_\mathrm{DPD}}(t) \cdot 10^{\beta / 20} \text {. }
$$

Substituting (6) into (5), PA ${ }_{j}$ 's output after the pre-distortion, $y_{j} \_$DPD $(t)$, can be written as

$$
\begin{aligned}
& y_{j \_\mathrm{DPD}}(t) \\
& \approx G_{j} \cdot g_{i}\left(x_{i \_\mathrm{DPD}}(t) \cdot 10^{\beta / 20} \cdot 10^{-\beta / 20}\right) \\
& =G_{j} \cdot g_{i}\left(x_{i \_\mathrm{DPD}}(t)\right) \\
& \left.=G_{j} \cdot x_{i}(t)\right),
\end{aligned}
$$

where $G_{j}$ is a constant, then $\mathrm{PA}_{j}$ is linearized.

The two terms $10^{\beta / 20}$ and $10^{-\beta / 20}$ from (7) are mutually eliminated. It also means that $\mathrm{PA}_{j}$ can be linearized by $\mathrm{PA}_{i}{ }^{\prime}$ s pre-distortion signal $x_{i \_ \text {DPD }}(t)$. Meanwhile, the output power of $\mathrm{PA}_{i}$ is $\alpha \mathrm{dB}$ higher than that of $\mathrm{PA}_{j}$. When the relationship demonstrated by (7) is satisfied between different operating modes, a cross-mode universal digital pre-distortion (CMUDPD) is achieved, which means that $\mathrm{PA}_{i}$ and $\mathrm{PA}_{j}$ have similar nonlinear characteristics, and the pre-distortion signal for each PA can be used to predistort each other. It should be noted that the shape function $g_{i}(\cdot)$ can be realized by any PA model, e.g., memory polynomial (MPM), general MPM, neural networks, etc. [38]. In this article, the popular MPM PA model is used.

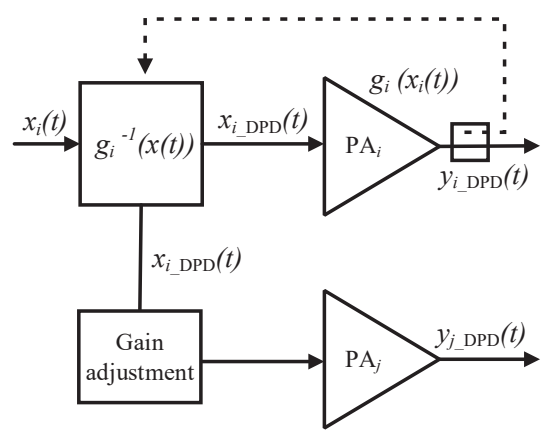

(a)

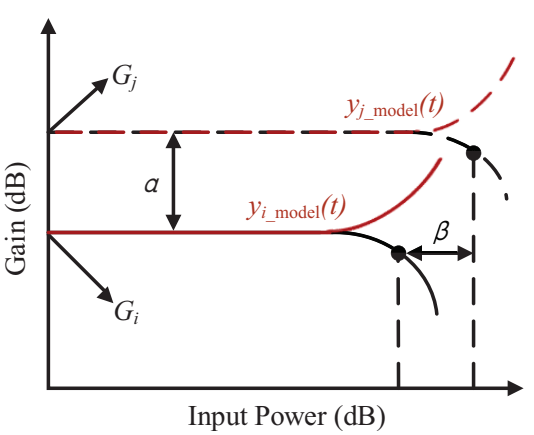

(b)

Figure 3. (a) Simulated circuit example used to illustrate theoretical principle of the proposed CMUDPD technique. (b) Gain curve versus input power in different PA operation modes.

\section{Simulation Validation}

The power amplifiers operating at different modes was studied through a system simulation using the Advanced Design System (ADS) and MATLAB ${ }^{\circledR}$. The schematic diagram of the simulation circuit is illustrated in Figure 4. A commercially available GaN PA, i.e., CGH40006P from CREE [39], was chosen for the validation as it allows operation in both Class $\mathrm{A}$ and Class $\mathrm{AB}$ with a wide range of supply and bias voltages. This provides benefits to thoroughly validate the proposed technique through a broad range of operation modes (Class AB and Class A cases) [40]. Cases with various combinations of supply voltage $V_{\mathrm{DD}}$ and bias voltage $V_{\mathrm{GG}}$ were simulated. According to the suggestion in the data sheet of CGH40006P [39], three supply voltages (i.e., $V_{\mathrm{DD}}=28,20$, and $12 \mathrm{~V}$ ) and five bias voltages (i.e., $V_{\mathrm{GG}}=-3.1,-2.9,-2.7,-2.5$ and $-2.3 \mathrm{~V}$ ) were chosen in the simulation cases. The ADS-obtained AM/AM curves with $V_{\mathrm{GG}}=28,20$, and $12 \mathrm{~V}$ are exhibited in Figure $5 \mathrm{a}-\mathrm{c}$, respectively. The AM/PM curves with $V_{\mathrm{GG}}=28,20$ and $12 \mathrm{~V}$ are presented in Figure $5 \mathrm{~d}-\mathrm{f}$, respectively. 


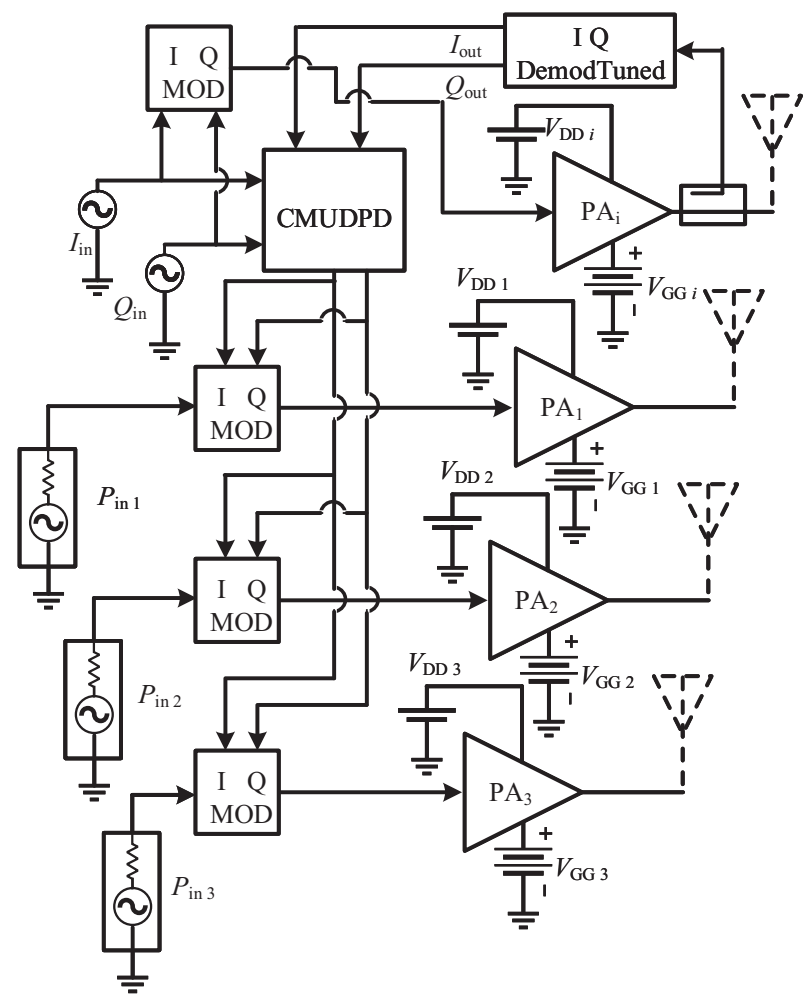

Figure 4. Schematic diagram of the simulation circuit.

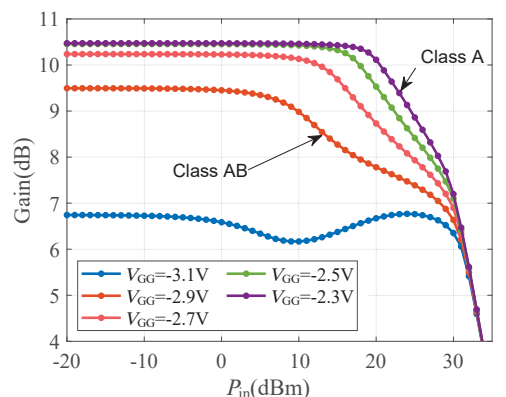

(a)

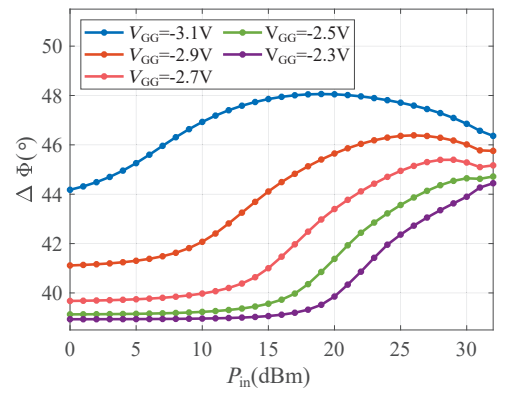

(d)

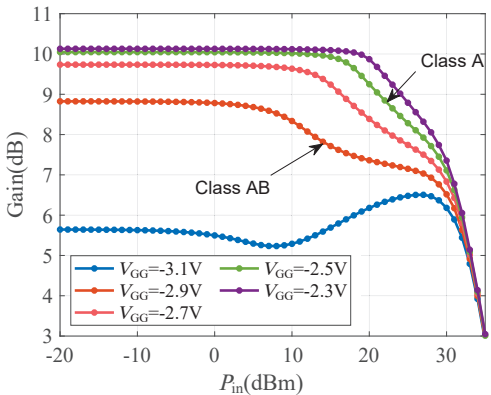

(b)

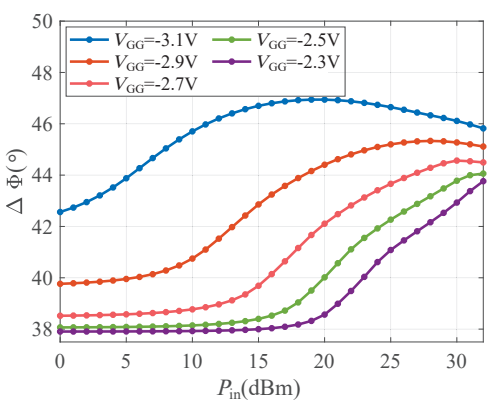

(e)

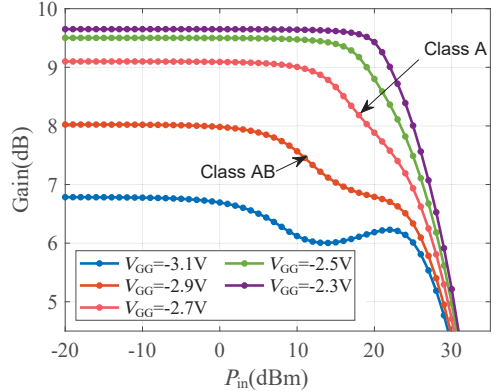

(c)

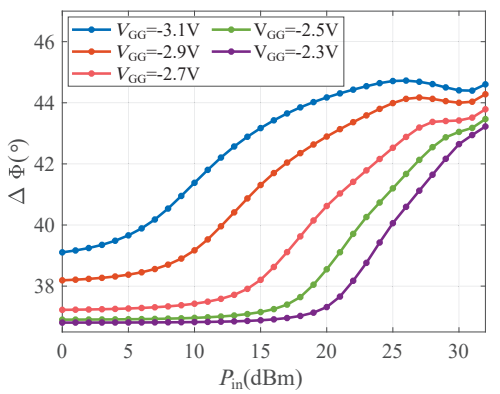

(f)

Figure 5. Advanced Design System (ADS) simulated AM/AM curves of the GaN PA: (a) $V_{\mathrm{DD}}=28 \mathrm{~V}$, (b) $V_{\mathrm{DD}}=20 \mathrm{~V}$, (c) $V_{\mathrm{DD}}=12 \mathrm{~V}$, and AM/PM curves; (d) $V_{\mathrm{DD}}=28 \mathrm{~V}$, (e) $V_{\mathrm{DD}}=20 \mathrm{~V}$, and (f) $V_{\mathrm{DD}}=12 \mathrm{~V}$.

Based on the different operation modes, the validation can be categorized into Group A for class AB operation modes and Group B for class A operation modes. In Group A, a constant $V_{\mathrm{GG}}(-2.9 \mathrm{~V})$ and varied $V_{\mathrm{DD}}(28,20$, and $12 \mathrm{~V})$ are chosen; in Group $\mathrm{B}$, variations 
in both $V_{\mathrm{GG}}(-2.3,-2.5$, and $-2.7 \mathrm{~V})$ and $V_{\mathrm{DD}}(28,20$, and $12 \mathrm{~V})$ are applied. Then, in the next step, the CMUDPD simulations were conducted based on the two groups, as mentioned above. A $10 \mathrm{MHz}$ LTE signal with a carrier frequency of $3.5 \mathrm{GHz}$ was used as the input signal. The average input power $P_{\text {in }}$ was set to the $1 \mathrm{~dB}$ compression point of the selected modes. The error vector magnitude (EVM) and adjacent channel power ratio $(\mathrm{ACPR})$ were used to evaluate the in-band and out-band distortions, respectively. The definition of EVM is given by

$$
\mathrm{EVM}=\sqrt{\frac{P_{\text {error }}}{P_{\text {reference }}}} \times 100 \%,
$$

where $P_{\text {error }}$ represents the average power of the error vector and $P_{\text {reference }}$ represents the average power of the reference signal [27]. The ACPR is defined as

$$
\mathrm{ACPR}=\frac{P_{\text {adjacent }}}{P_{\text {main }}},
$$

where $P_{\text {main }}$ is the main channel power, and $P_{\text {adjacent }}$ is the average power of the lower adjacent channel and upper adjacent channel [41]. The simulated drain efficiency values are shown in Table 2, where the values were calculated as an average of 10,000 samples. It can be seen that the drain efficiencies of all studied operation modes improved after the CMUDPD. The detailed simulation procedures were as follows:

- Step 1: Drive the PAs to one of the different operation modes while working at the $1 \mathrm{~dB}$ compression point.

- $\quad$ Step 2: Select an operation mode $i$ in the low-sidelobe active antenna array, as shown in Figure 2, to obtain the output signal for pre-distortion.

- Step 3: According to the output signal obtained in step 2, model the PAs in the array in MATLAB. Collect the coefficient of CMUDPD using MPM, and the obtain pre-distorted input signal.

- $\quad$ Step 4: Load the pre-distorted signal into the CMUDPD block of ADS. Then change the PA's operating mode according to Table 2. After the CMUDPD, obtain the linearized outputs of PAs.

- $\quad$ Step 5: Plot the normalized power spectral density (PSD) and calculate EVM and ACPR.

Table 2. Different modes setup of Group A (mode a1, mode a2, and mode a3) and Group B (mode b1, mode b2, and mode b3). Format of the drain efficiency (eff.) column: without CMUDPD and with CMUDPD.

\begin{tabular}{cccccc}
\hline Mode $\boldsymbol{i}$ & $\boldsymbol{P}_{\text {in }}$ & $\boldsymbol{V}_{\text {DD }}$ & $\boldsymbol{V}_{\mathrm{GG}}$ & Drain Eff. (\%) & $\boldsymbol{\varepsilon}(\mathbf{d B})$ \\
\hline mode a1 & $13.6 \mathrm{dBm}$ & $28 \mathrm{~V}$ & $-2.9 \mathrm{~V}$ & $14.54 / 20.23$ & -54.84 \\
mode a2 & $14.0 \mathrm{dBm}$ & $20 \mathrm{~V}$ & $-2.9 \mathrm{~V}$ & $17.81 / 30.50$ & -53.82 \\
mode a3 & $15.3 \mathrm{dBm}$ & $12 \mathrm{~V}$ & $-2.9 \mathrm{~V}$ & $34.15 / 38.13$ & -52.40 \\
mode b1 & $23.3 \mathrm{dBm}$ & $28 \mathrm{~V}$ & $-2.3 \mathrm{~V}$ & $42.48 / 43.11$ & -50.76 \\
mode b2 & $21.0 \mathrm{dBm}$ & $20 \mathrm{~V}$ & $-2.5 \mathrm{~V}$ & $27.45 / 29.64$ & -47.63 \\
mode b3 & $18.2 \mathrm{dBm}$ & $12 \mathrm{~V}$ & $-2.7 \mathrm{~V}$ & $18.04 / 22.98$ & -45.45 \\
\hline
\end{tabular}

In step 3, a traditional MPM was used to model the power amplifier, which can be expressed as follows:

$$
y(n)=\sum_{m=0}^{M} \sum_{k=1}^{K} a_{m k} x(n-m)|x(n-m)|^{k-1}
$$


where $M, K$, and $a_{m k}$ denote the memory depth, the order of memory polynomial, and the coefficient of memory polynomial, respectively. Assuming that the length of the input original standard signal data stream $x(n)$ is $L$, the linear gain of PA can be written as

$$
G_{\text {linear }}=\left\{\sum_{n=1}^{L}|y(n) \cdot / x(n)|\right\} / L .
$$

Then, the input signal for training, $X_{\text {train }}$, can be written as

$$
X_{\text {train }}=\left[\begin{array}{llll}
x(M) & x(M+1) & \cdots & x(L)
\end{array}\right] .
$$

The output signal of (10) can be written as an array $Y$ that is amplified by the power amplifier with a memory depth of $M$ :

$$
\begin{aligned}
& Y=\left[\begin{array}{llll}
Y_{M} & Y_{M-1} & \cdots & Y_{1}
\end{array}\right], \\
& Y_{i}=\left[\begin{array}{c}
y(:, i) \\
y(:, i) \cdot y(:, i) \\
\vdots \\
y(:, i) \cdot y(:, i)(K-1)
\end{array}\right]^{T},
\end{aligned}
$$

where $i \in(1: M)$ and $y(:, i)$ represent the $i$ th column of the output matrix as exhibited in (15),

$$
y(:, i)=\left[\begin{array}{c}
y(i) \\
y(i+1) \\
\vdots \\
y(L-M+i)
\end{array}\right]^{T} .
$$

Based on (12) and (13), the coefficient matrix of the obtained memory polynomial inversion can be written as

$$
A=Y \backslash X_{\text {train }}
$$

Furthermore, the model input obtained by inverting the MPM is calculated as

$$
X_{\text {model }}=Y * A .
$$

The error between the model input $X_{\text {model }}$ and the trained input $X_{\text {train }}$ reflects the accuracy of the memory polynomial modeling of the PA. In order to quantify the error, a mean square error $\varepsilon$ is introduced by

$$
\varepsilon=10 \cdot \log _{10}\left\{\left(X_{\text {model }}-X_{\text {train }}\right)^{2} /(L-M) / X_{\text {train }}{ }^{2}\right\} .
$$

When $\varepsilon$ reaches the required linearization requirement, the output model of the PA is obtained. Subsequent simulations and experiments showed that when $\varepsilon$ reaches approximately $-45 \mathrm{~dB}$, the linearization performance meets the satellite communication linearity requirements [42-44]. The effect of CMUDPD is highly dependent on the accuracy of the PA model.

$$
Y_{\text {model }}=X_{\text {model }} * G_{\text {linear }},
$$


where $G_{\text {linear }}$ denotes the linear gain of the PA, as defined in (11). Then, the pre-distorted input signal of the PA can be obtained by

$$
X_{\mathrm{MPM}}=Y_{\text {model }} * A \text {. }
$$

Bringing $X_{\mathrm{MPM}}$ into PA produces a linear convergence gain $G_{\mathrm{CMUDPD}}$, as shown in Figure 6, where $G_{\text {model }}$ can be represented by $X_{\mathrm{MPM}}$ as

$$
G_{\text {model }}=X_{\mathrm{MPM}} / X_{\text {train }} * G_{\text {linear }} \text {. }
$$

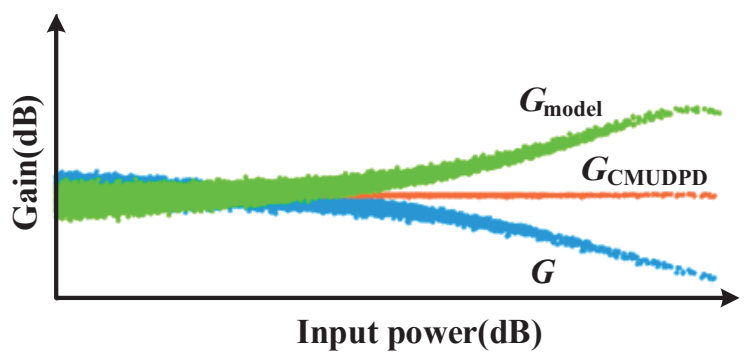

Figure 6. The gain compensation of CMUDPD.

The output PSDs of all operation modes obtained by ADS simulations are shown in Figure 7 (i.e., a1 W/O DPD, a2 W/O DPD, a3 W/O DPD, b1 W/O DPD, b2 W/O DPD, and b3 W/O DPD). For comparison, Figure 7 also depicts the output PSDs of all nonlinear models calculated by MATLAB (a1 model, a2 model, a3 model, b1 model, b2 model, and b3 model). It can be seen that different operation modes for each group (i.e., modes a1, a2, and a3 for Group A; and modes b1, b2, and b3 for Group B) exhibit insignificant PSD differences. Thereby, the parameter $\varepsilon$ defined in (18) can be utilized to identify the degree of similarity in terms of nonlinearities among different operation modes.

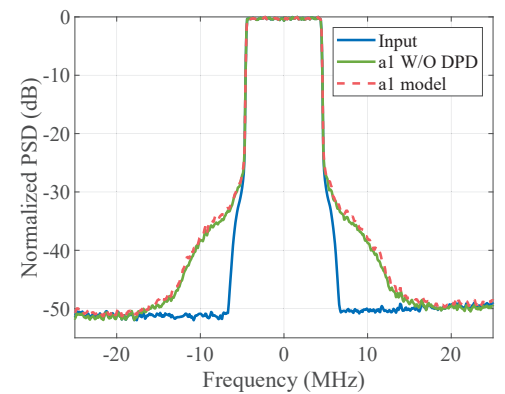

(a)

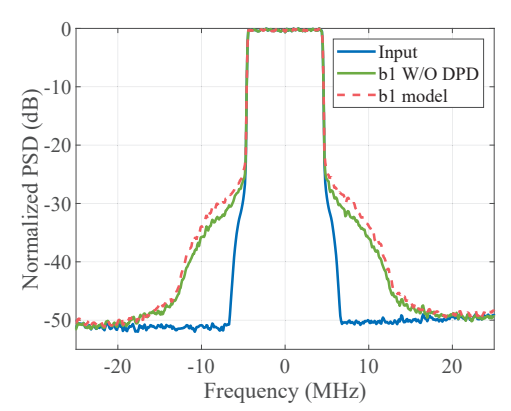

(d)

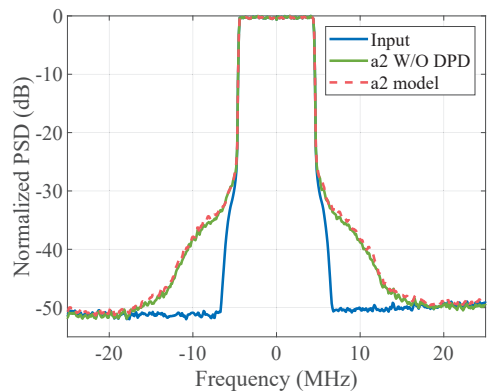

(b)

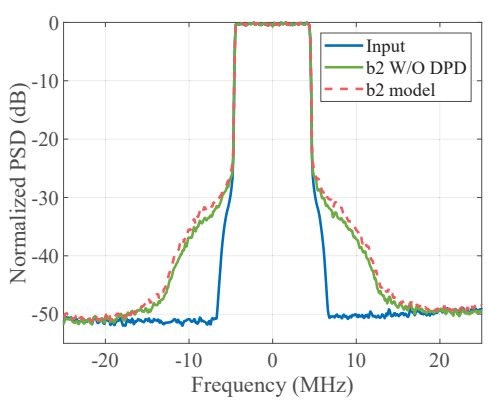

(e)

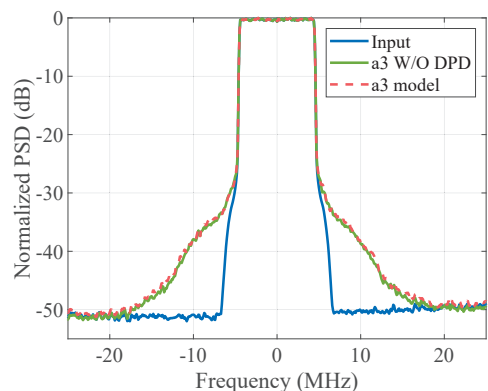

(c)

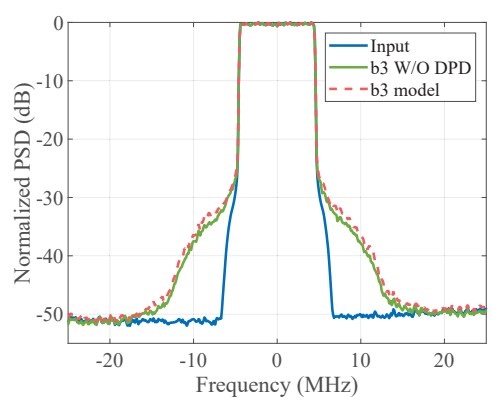

(f)

Figure 7. Simulated results of the output PSDs. Group A: (a) mode a1, (b) mode a2, and (c) mode a3. Group B: (d) mode b1, (e) mode b2, and (f) mode b3. 


\subsection{Group A: CMUDPD of PAs Operating in Class $A B$}

In this group, the PAs are working in Class $\mathrm{AB}$ with a constant bias voltage $V_{\mathrm{GG}}$ $(-2.9 \mathrm{~V})$ and varied supply voltage $V_{\mathrm{DD}}(28,20$, and $12 \mathrm{~V})$, as shown in Figure 8 . The $1 \mathrm{~dB}$ compression point (indicated by arrows) of these three gain curves was selected as the input power of each mode. At first, the PAs containing operation modes a1, a2, and a3 were simulated by ADS, and the output signal before pre-distortion was obtained. By injecting these output signals into MATLAB, the coefficients $a_{m k}$ of CMUDPD were obtained through the memory polynomial $(M=7, K=5)$. Then, the pre-distortion signal obtained by inverting the MPM was loaded into the CMUDPD block. Finally, the PSDs with and without CMUDPD1, CMUDPD2, and CMUDPD3 are shown in Figure 9a-c, respectively, where CMUDPD1, CMUDPD2, and CMUDPD3 represent the pre-distortion results when the outputs of mode a1, a2, and a3 are used as the feedback paths, respectively.

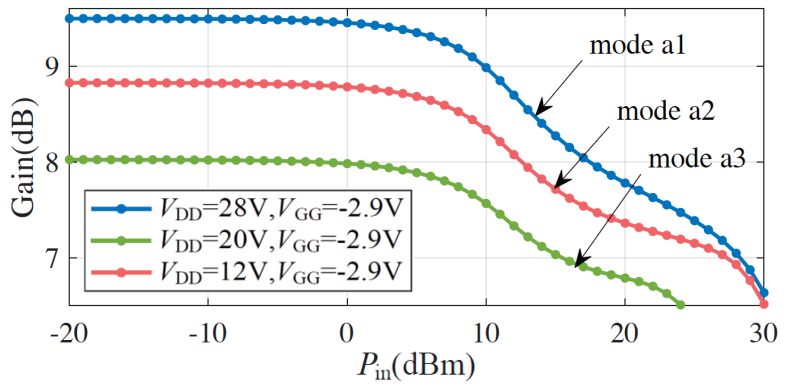

(a)

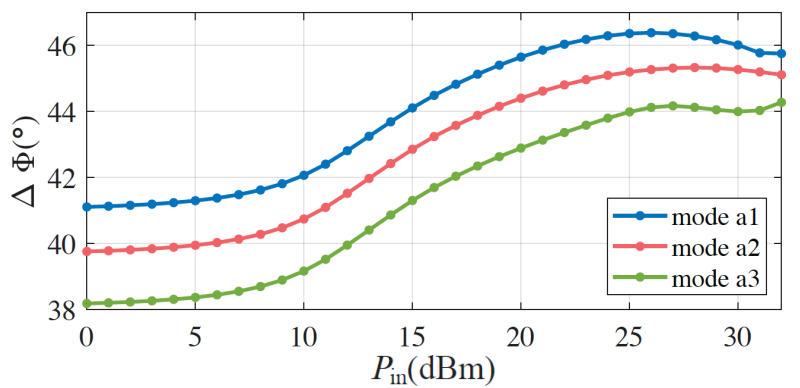

(b)

Figure 8. Nonlinear characteristics of Class AB. (a) Gain curves versus input power at Class AB. (b) AM/PM characteristic of Class AB.

Figure 9a shows that the pre-distortion signals generated by mode a1 can successfully linearize modes a1, a2, and a3. Similarly, the pre-distortion signals from modes a2 and a3 can also be used to linearize all these three modes, which can be concluded from Figure $9 \mathrm{~b}, \mathrm{c}$. It can be obtained from Table 2 that, in group $\mathrm{A}$, the mean square errors $\varepsilon$ of the three operation modes are comparable. The memory polynomial $(M=7, K=5)$ can fit any of the three modes, and any of the three modes can be used as the feedback mode to extract the pre-distortion input for the whole group of modes. The best pre-distortion performance for the three operation modes in Group A can be obtained when mode a2 is used as the feedback mode. This can be explained by the $\varepsilon$ value of mode a2 $(-53.82 \mathrm{~dB})$ being in between these values of modes a1 $(-52.40 \mathrm{~dB})$ and a3 $(-54.84 \mathrm{~dB})$. 


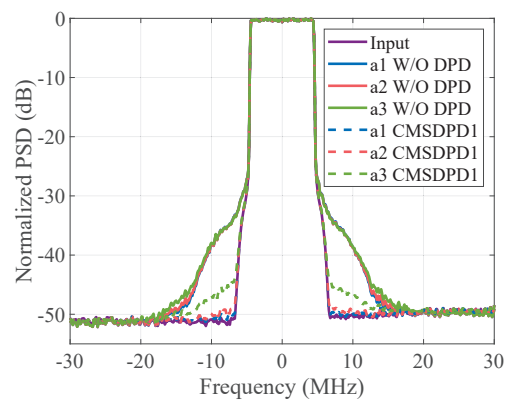

(a)

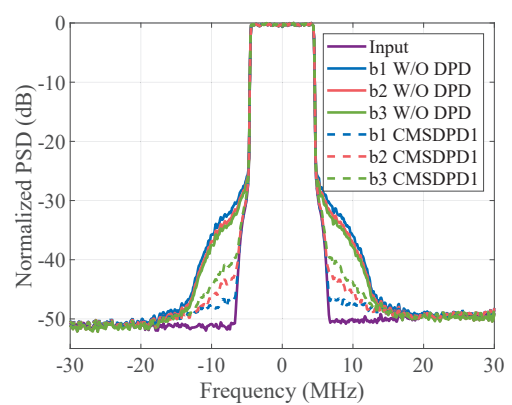

(d)

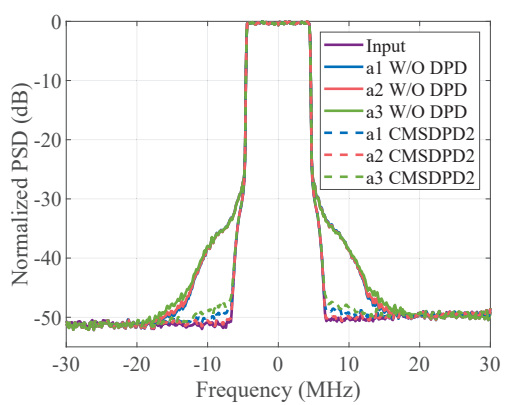

(b)

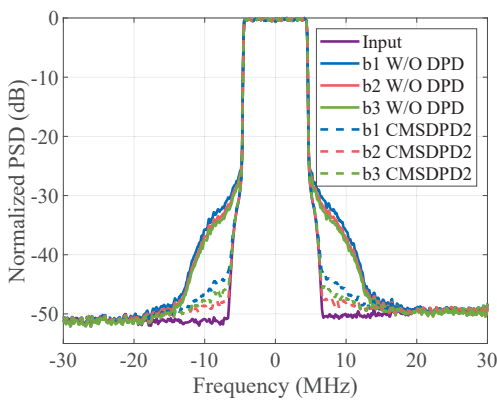

(e)

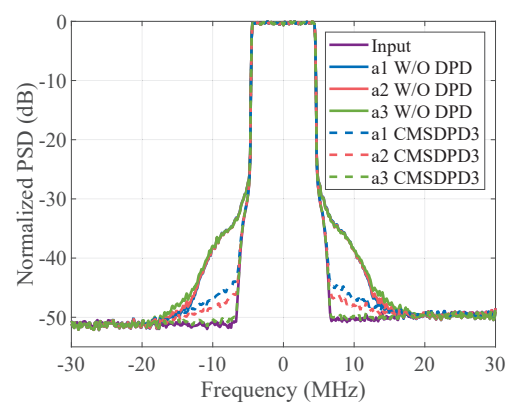

(c)

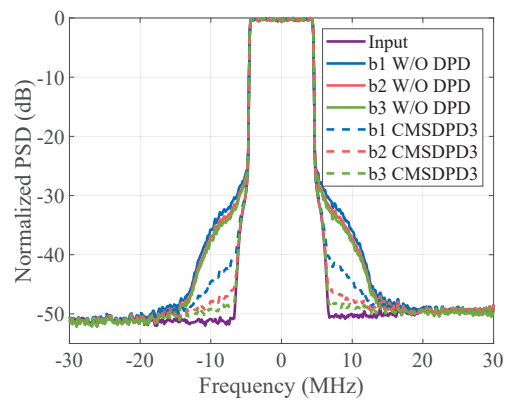

(f)

Figure 9. Simulation results of PSDs with and without CMUDPD. (a) Modes a1, a2, and a3 with and without CMUDPD1. (b) Modes a1, a2, and a3 with and without CMUDPD2. (c) Modes a1, a2, and a3 with and without CMUDPD3. (d) Modes b1, b2, and b3 with and without CMUDPD1. (e) Modes b1, b2, and b3 with and without CMUDPD2. (f) Modes b1, b2, and b3 with and without CMUDPD3.

\subsection{Group B: CMUDPD of PAs Operating in Class $A$}

As shown in Figure 10, Group B has both varying supply voltages $V_{\mathrm{DD}}(28,20$, and $12 \mathrm{~V})$ and bias voltages $V_{\mathrm{GG}}(-2.3,-2.5$, and $-2.7 \mathrm{~V})$. The input powers of the corresponding modes are set at the $1 \mathrm{~dB}$ compression point (indicated by arrows). The similarities in the nonlinearities among the three modes are indicated by $\varepsilon$ in Table 2. Compared with Group A, the values of $\varepsilon$ in Group B are relatively higher. Nevertheless, this does not significantly affect the pre-distortion performance of CMUDPD. In Group B, the PAs are in higher output power and greater bias voltage, so there is a higher probability for third-order inter-modulation compared to Group A. In group B, the memory polynomial $(M=7, K=3)$ can fit any of the three modes, and any of the three modes can be used as a feedback mode to extract the pre-distortion input for the entire mode group.

The simulated linearization performances of Group B are depicted in Figure $9 d-f$, where CMUDPD1, CMUDPD2, and CMUDPD3 represent the pre-distortion results when the outputs of mode b1, b2, and b3 are used as the feedback paths, respectively. An analogous conclusion can also be drawn for Group B, where using b2 as the feedback mode produces superior pre-distortion performance. Group A's overall linearization performance is superior to that in Group B since the values of $\varepsilon$ in Group A are lower than those in Group $B$. This indicates that the overall pre-distortion effects are determined by the accuracy of the memory polynomial model.

\subsection{Comparison between Group A and Group B}

First, the differences between the simulation setup are shown in Table 2. For Group $\mathrm{A}$, the bias voltage is constant and the supply voltage is varied. The operation modes are different, but they all belong to Class AB. For Group B, both bias voltage and supply voltage are varied, with more significant differences between the operating modes. By adjusting the bias voltage and supply voltage, the PA will operate in Class AB or Class 
A. Then, the nonlinear characteristics of Class $A B$ and $A$ are shown in Figures 8 and 10. Figure $8 \mathrm{a}, \mathrm{b}$ depicts the $\mathrm{AM} / \mathrm{AM}$ curves and $\mathrm{AM} / \mathrm{PM}$ curves of Class $\mathrm{AB}$, respectively. Moreover, Figure 10a,b is the AM/AM curves and AM/PM curves of Class A, respectively. As can be seen from Figure $8 \mathrm{~b}$, the AM/PM curve shapes among modes a1, a2, and a3 are similar. Nevertheless, in Figure 10b, the AM/PM curves of Class A cross each other under a larger input power $P_{\text {in }}$.

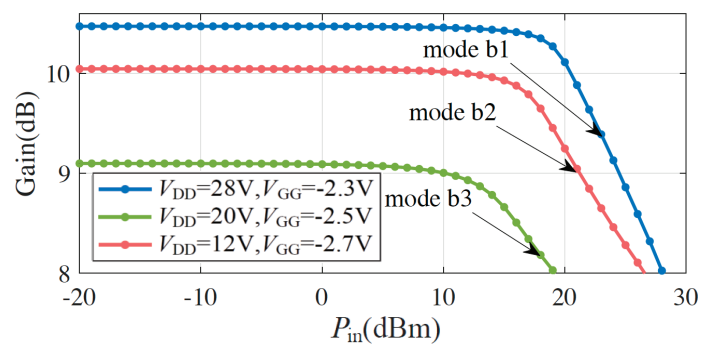

(a)

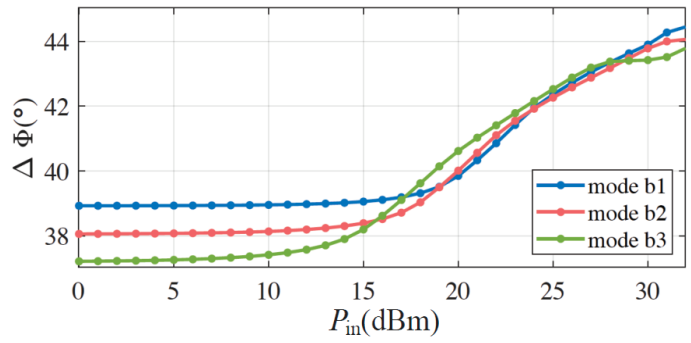

(b)

Figure 10. Nonlinear characteristics of Class A. (a) Gain curves versus input power for Class A. (b) AM/PM characteristic of Class A.

It can be concluded from Figure 9b,e that the normalized PSDs with the CMUDPDs (a2 and $\mathrm{b} 2$ as feedback modes) can be suppressed by $13 \mathrm{~dB}$ on average. It can also be seen from Figure 11a-d that AM/AM and AM/PM with CMUDPDs become significantly convergent compared to those without CMUDPDs. Moreover, the results using an arbitrary mode as feedback to linearize other modes are summarized in Table 3. Other modes with CMUDPD, e.g., a1, a3, b1, and b3, can also achieve ACPR suppressions above 11 dB. For both Group $A$ and Group B, the values of the error vector magnitudes (EVMs) with CMUDPD are reduced by one order of magnitude compared to those without CMUDPDs.

Table 3. The simulation (simu.) and experimental (expt.) results of CMUDPD in Group A and Group B with each of the three modes as feedback modes.

\begin{tabular}{cccccc}
\hline Group A & $\begin{array}{c}\text { EVM } \\
\text { Simu./Expt. }\end{array}$ & $\begin{array}{c}\text { ACPR (dB) } \\
\text { Simu./Expt. }\end{array}$ & Group B & $\begin{array}{c}\text { EVM } \\
\text { Simu./Expt. }\end{array}$ & $\begin{array}{c}\text { ACPR (dB) } \\
\text { Simu./Expt. }\end{array}$ \\
\hline mode a1 W/O DPD & $5.20 \% / 5.90 \%$ & $-35.86 /-38.52$ & mode b1 W/O DPD & $6.79 \% / 8.23 \%$ & $-32.90 /-32.32$ \\
mode a2 W/O DPD & $5.21 \% / 6.04 \%$ & $-35.99 /-38.53$ & mode b2 W/O DPD & $5.93 \% / 8.34 \%$ & $-34.15 /-32.02$ \\
mode a3 W/O DPD & $5.31 \% / 7.18 \%$ & $-36.07 /-38.23$ & mode b3 W/O DPD & $5.57 \% / 8.21 \%$ & $-34.97 /-32.01$ \\
mode a1 W CMUDPD1 & $0.54 \% / 0.78 \%$ & $-49.13 /-49.21$ & mode b1 W CMUDPD1 & $0.76 \% / 0.93 \%$ & $-47.28 /-48.85$ \\
mode a2 W CMUDPD1 & $0.64 \% / 0.90 \%$ & $-49.12 /-49.38$ & mode b2 W CMUDPD1 & $1.54 \% / 1.00 \%$ & $-45.59 /-48.91$ \\
mode a3 W CMUDPD1 & $1.21 \% / 1.04 \%$ & $-46.58 /-48.75$ & mode b3 W CMUDPD1 & $2.14 \% / 1.28 \%$ & $-43.67 /-43.95$ \\
mode a1 W CMUDPD2 & $0.51 \% / 0.59 \%$ & $-49.13 /-49.27$ & mode b1 W CMUDPD2 & $1.47 \% / 0.98 \%$ & $-45.91 /-48.95$ \\
mode a2 W CMUDPD2 & $0.50 \% / 0.66 \%$ & $-49.44 /-48.86$ & mode b2 W CMUDP2 & $0.60 \% / 0.87 \%$ & $-48.08 /-49.02$ \\
mode a3 W CMUDPD2 & $0.74 \% / 0.96 \%$ & $-48.21 /-49.27$ & mode b3 W CMUDPD2 & $0.87 \% / 1.26 \%$ & $-47.05 /-49.06$ \\
mode a1 W CMUDPD3 & $1.18 \% / 1.23 \%$ & $-46.42 /-47.26$ & mode b1 W CMUDPD3 & $1.94 \% / 3.01 \%$ & $-43.39 /-43.07$ \\
mode a2 W CMUDPD3 & $0.64 \% / 0.74 \%$ & $-49.84 /-45.52$ & mode b2 W CMUDPD3 & $0.86 \% / 0.89 \%$ & $-47.56 /-49.14$ \\
mode a3 W CMUDPD3 & $0.82 \% / 0.87 \%$ & $-49.74 /-49.26$ & mode b3 W CMUDPD3 & $0.52 \% / 0.66 \%$ & $-48.54 /-49.18$ \\
\hline
\end{tabular}




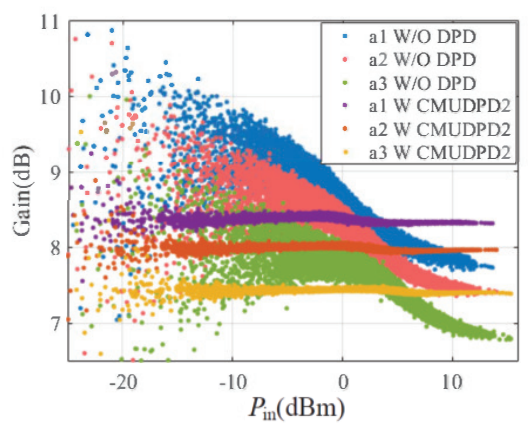

(a)

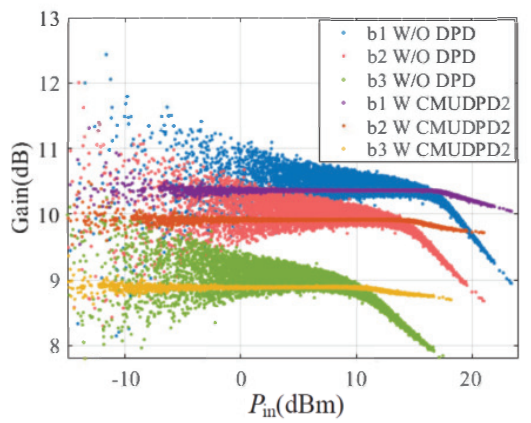

(c)

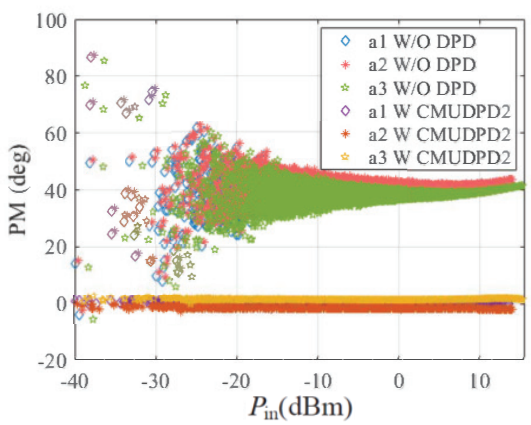

(b)

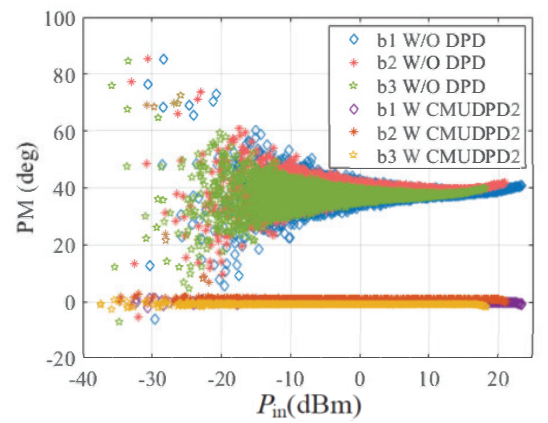

(d)

Figure 11. Simulated AM/AM and AM/PM curves. (a) Group A's AM/AM curves with (W) and without (W/O) CMUDPD2. (b) Group A's AM/PM curves W and W/O CMUDPD2. (c) Group B's AM/AM curves W and W/O CMUDPD2. (d) Group B's AM/PM curves W and W/O CMUDPD2.

\section{Experimental Validation and Discussion}

\subsection{Experimental Setup and Results Analysis}

The experimental setup is shown in Figure 12. A computer was used to upload the base-band input signal to a signal generator (R\&S SMBV100A). Subsequently, the base-band I/Q output signals from the signal analyzer (R\&S FSQ26) were recorded by the computer. Table 3 gives the cases where all modes are used as the feedback operation modes. The experiment steps were as follows:

- Generate the input signal and record the output signal under different operation modes. Load an LTE signal with a bandwidth of $10 \mathrm{MHz}, x(n)$, from the computer to the signal generator. Excite the PA using the modulated signal and record the output, $y(n)$, from the signal analyzer to the computer.

- Calculate the pre-distorted signal by MPM. When testing Group A, generate the pre-distorted signal, $x_{\mathrm{DPD}}(n)$, by the MPM with a memory depth of 7 and an order of 5 using the recorded $x(n)$ and $y(n)$. When testing Group B, generate the pre-distorted signal, $x_{\mathrm{DPD}}(n)$, by the MPM with a memory depth of 7 and an order of 3 using the recorded $x(n)$ and $y(n)$.

- Load the pre-distorted signal $x_{\mathrm{DPD}}(n)$ from the computer into the signal generator and excite the PA using $x_{\mathrm{DPD}}(n)$ under different modes. Record the corresponding pre-distorted output signals and calculate PSD, EVM, and ACPR.

When testing and verifying Group $\mathrm{A}, V_{\mathrm{DD}}$ was set to 28,20 , and $12 \mathrm{~V}$, and the input power level iwass selected as the $1 \mathrm{~dB}$ compression point, which was calculated based on the simulation. The performance metrics of the experimental linearization, i.e., EVM and ACPR, are also listed in Table 3. It can be seen that the experimental results satisfactorily match the simulations. For both groups, the experimental EVM after the CMUDPD reduced by an order of magnitude. The experimental ACPR is lower than $-45 \mathrm{~dB}$ on average, and an averaged ACPR suppression of $13 \mathrm{~dB}$ was achieved. As CMUDPD2 achieved superior 
performance for both groups in Table 3, the experimental PSDs with CMUDPD2 for Group $\mathrm{A}$ and $\mathrm{B}$ are demonstrated in Figure 13a,b, respectively. The pre-distortion performance is consistent with the simulation results. As shown in Figure 13b, the proposed CMUDPD provides effective linearization performance even under severe out-band distortion conditions.

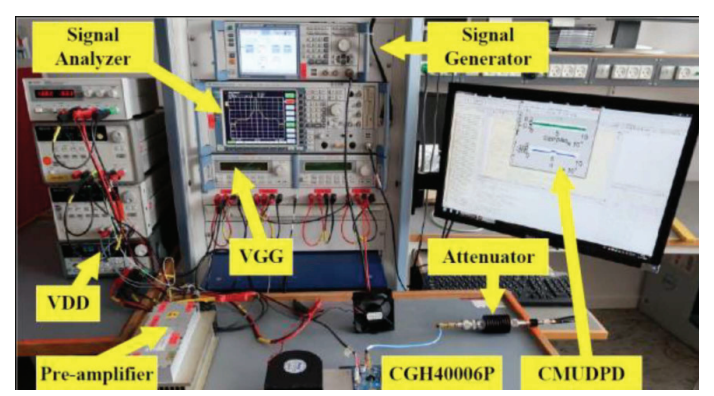

Figure 12. Experiment setup.

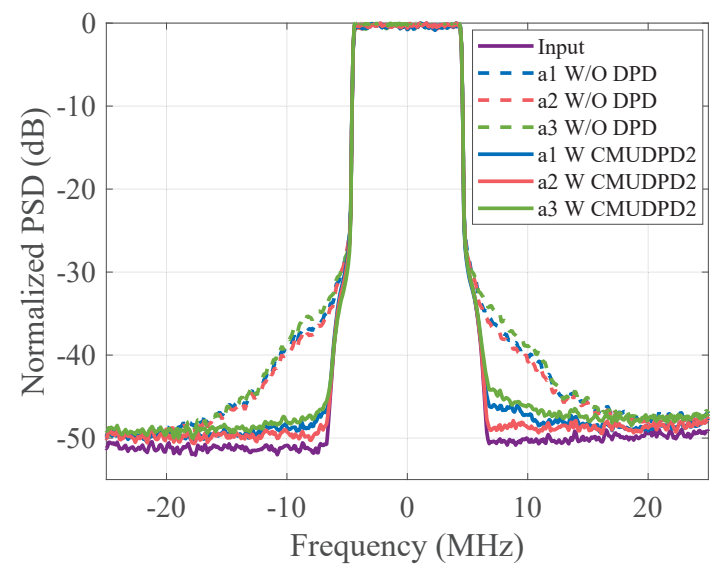

(a)

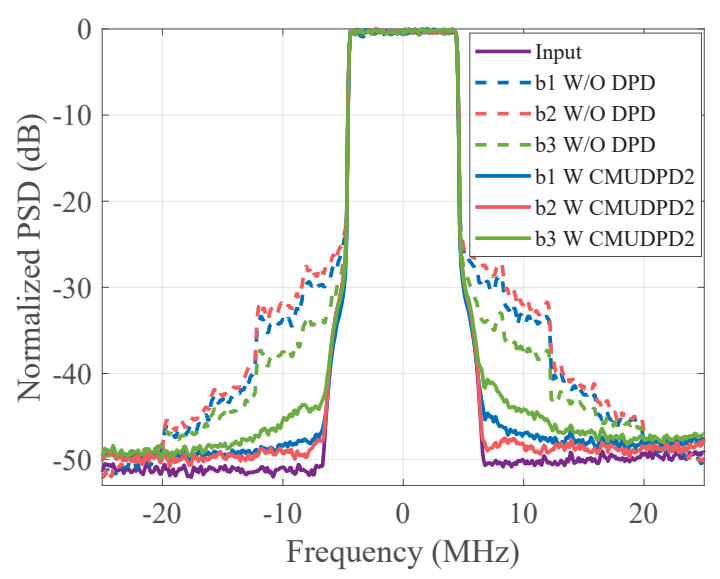

(b)

Figure 13. Experimental results of PSDs with (W) and without (W/O) CMUDPD2. (a) Group A: mode a1, a2, and a3 W and W/O CMUDPD2. (b) Group B: mode b1, b2, and b3 W and W/O CMUDPD2.

In order to strengthen the usefulness of the proposed CMUDPD, the experiment setup with multiple PAs was built as exhibited in Figure 14. Four identical PAs were used, denominated as $\mathrm{PA}_{1}, \mathrm{PA}_{2}, \mathrm{PA}_{3}$, and $\mathrm{PA}_{4}$ from left to right. A ventilation fan was installed on the heatsink to dissipate the heat generated by the PAs. Due to the limitation due to the unequal power divider lacking in the laboratory, it was not possible to accurately feed different input powers to the PA array in a single trial. Nevertheless, an equal power 
divider was implemented in the experiment to excite the PAs, and the different input power levels were achieved by multiple trials. First, the output of $\mathrm{PA}_{1}$ (operating in mode a1) was recorded from the signal analyzer. The pre-distorted signal was calculated by the aforementioned MPM method. Then, the pre-distorted signal was loaded to the signal generator, which in turn excited $\mathrm{PA}_{2}, \mathrm{PA}_{3}$, and $\mathrm{PA}_{4}$, which operated in mode $\mathrm{a} 1, \mathrm{a} 2$, and a3, respectively. The experimental PSD results are shown in Figure 15, where $\mathrm{PA}_{2}$ with CMUDPD exhibits superior performance due to operating in the identical mode as $\mathrm{PA}_{1}$. It can be concluded from Figure 15 that $\mathrm{PA}_{2}, \mathrm{PA}_{3}$, and $\mathrm{PA}_{4}$ with CMUDPD exhibit satisfactory results considering the manufacturing tolerance of practical PA products.

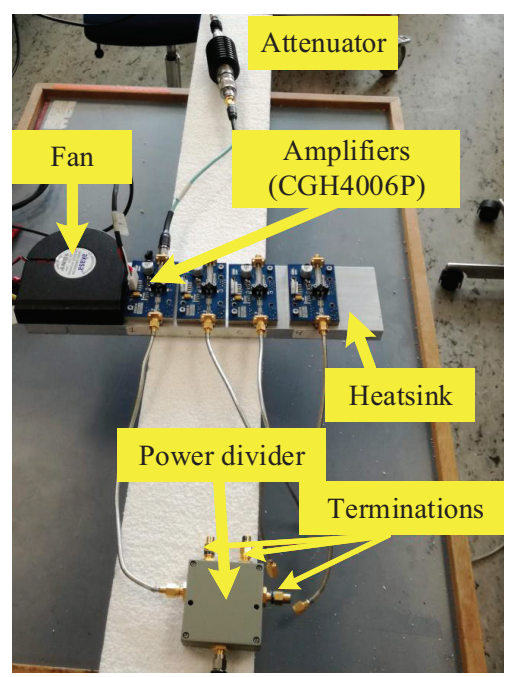

Figure 14. The experiment setup with multiple PAs.

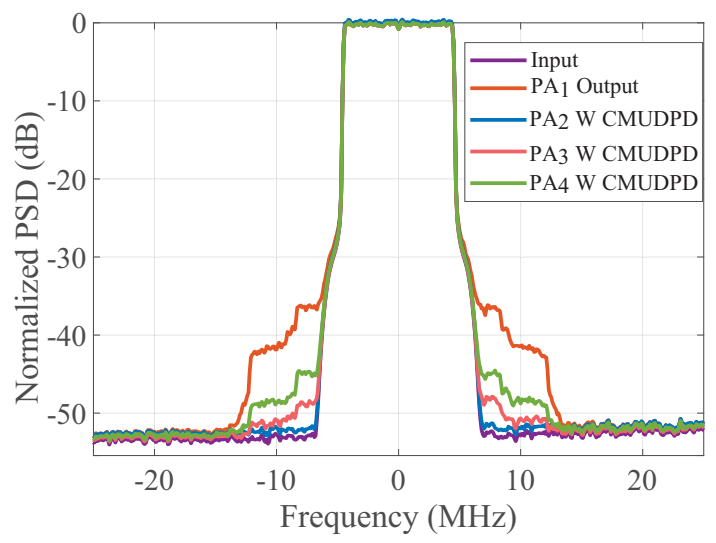

Figure 15. Experimental results of PSD with (W) CMUDPD.

\subsection{Wide Band Validation}

The validation of the proposed CMUDPD technique with $30 \mathrm{MHz}$ OFDM signals was also conducted. The PSD results (with and without CMUDPD) are depicted in Figure 16a,b, where CMUDPD1, CMUDPD2, and CMUDPD3 represent the pre-distortion results when the outputs of modes $\mathrm{a} 1 / \mathrm{b} 1, \mathrm{a} 2 / \mathrm{b} 2$, and $\mathrm{a} 3 / \mathrm{b} 3$ wer used as feedback paths, respectively. It can be seen from Figure 16 that the proposed CMUDPD works well, achieving an ACPR reduction of $11 \mathrm{~dB}$ in Class $\mathrm{AB}$ and $13 \mathrm{~dB}$ in Class $\mathrm{A}$. Due to the limitation of the available LTE OFDM modulation signal templates and capability of uploading wide-band predistortion signals in the used signal generator, the validation was performed by simulation using the nonlinear equivalent circuit model of the same PA in the experimental validation. However, we think that the proposed technique has potential for even wider band signals, e.g., $100 \mathrm{MHz}$, which may be an interesting topic for future investigation. 


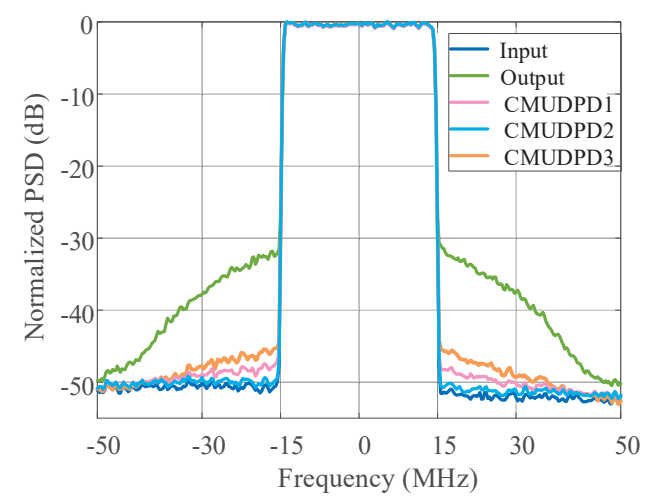

(a)

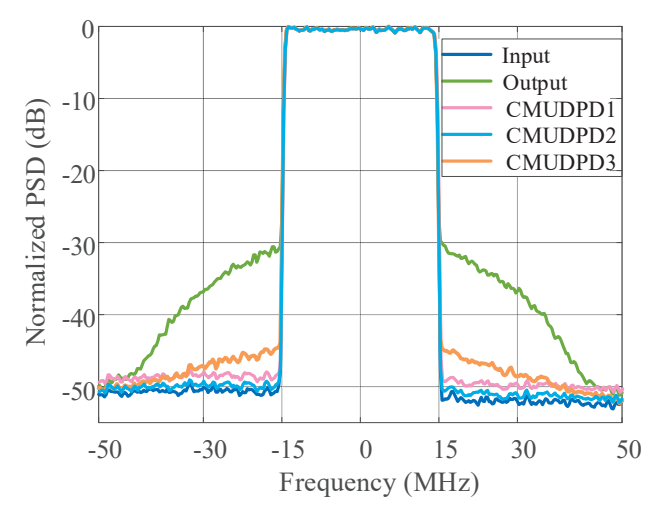

(b)

Figure 16. Simulation results of PSDs under an experimentally generated $30 \mathrm{MHz}$ bandwidth signal with (W) and without (W/O) CMUDPD. (a) Class AB W and W/O CMUDPD. (b) Class A W and W/O CMUDPD.

In this work, the used a CGH40006P PA from CREE, which features a frequency range of 2-6 GHz. Hence, the $3.5 \mathrm{GHz}$ center frequency was chosen for the experimental validation, and the peripheral impedance matching circuit was specifically designed based on $3.5 \mathrm{GHz}$. Nevertheless, the proposed CMUDPD method is not frequency-dependent, as can be seen in (7), and hence is applicable to other frequency ranges. Furthermore, the simulation and experimental validations were conducted using a GaN HEMT in this study. In future works, the proposed CMUDPD principle is expected to be applied to other types of transistors/PAs.

\subsection{The Drain Current Deviations Tolerance Capability of CMUDPD}

The PA operation modes in the actual antenna array system may involved several complicated situations, e.g., the PA is not a standard class AB or class A and has inconsistent operation modes. Therefore, an ADS simulation was conducted by manually introducing the drain current deviation to verify the tolerance capability of CMUDPD. The drain current deviation was caused by increasing the bias current by $10 \%$ or decreasing the bias current by $10 \%$. The three different operation modes' PSDs without and with CMUDPD are shown in Figure 17 (i.e., a1 W/O DPD, a2 W/O DPD, a3 W/O DPD, a1 W CMUDPD2, a2 W CMUDPD2, and a3 W CMUDPD2). For comparison, the light-colored lines represent the PSDs of the pre-distortion outputs with the drain current deviations (i.e., a1 W $\mathrm{I}_{\mathrm{D} 1} * 1.1$, a2 $\mathrm{WI}_{\mathrm{D} 2} * 1.1$, a $3 \mathrm{WI}_{\mathrm{D} 3} * 1.1$, a $1 \mathrm{~W} \mathrm{I}_{\mathrm{D} 1} * 0.9$, a $2 \mathrm{~W} \mathrm{I}_{\mathrm{D} 2} * 0.9$, a $\left.3 \mathrm{~W} \mathrm{I} \mathrm{D} 3 * 0.9\right)$. It can be seen that the CMUDPD can still reduce the PSD to below $-45 \mathrm{~dB}$. Even though the $\pm 10 \%$ drain current deviation was introduced, the proposed CMUDPD still exhibited satisfactory results. 


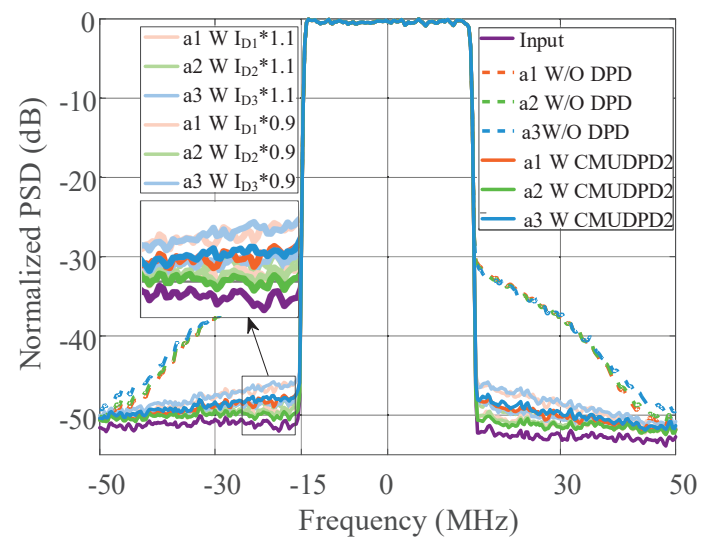

Figure 17. Normalized PSDs of different operation modes with drain current deviations $\pm 10 \%$ $\left(\mathrm{I}_{\mathrm{D} 1}=66.82 \mathrm{~mA}, \mathrm{I}_{\mathrm{D} 2}=61.20 \mathrm{~mA}, \mathrm{I}_{\mathrm{D} 3}=63.35 \mathrm{~mA}\right)$.

\section{Conclusions}

This paper revealed the possibility to achieve nonlinearity correlations between PAs operating in different saturation modes with different desired output power levels. Based on this finding, the main contribution, a cross-mode universal pre-distortion (CMUDPD) technique is proposed for the linearization of PAs in low-sidelobe active antenna arrays. With the achieved nonlinearity correlation, a single pre-distortion feedback path is sufficient for linearizing the PAs in the whole antenna array using the proposed technique. Extensive simulation and experimental validations were conducted covering a broad range of operation specifications, including two PA classes (i.e., Class A and Class AB) and two signal bandwidths (i.e., 10 and $30 \mathrm{MHz}$ ). The validation results showed that the proposed technique can successfully achieve an averaged ACPR suppression of $13 \mathrm{~dB}$ for multiple GaN PAs operating with different output power levels desired in low-sidelobe arrays. Compared with other existing linearization techniques, the proposed CMUDPD technology reduces the complexity of the system and the power consumption and hence shows the potential to be applied to satellite communication systems and 5G/6G networks, which exhibit stringent requirements on power efficiency and system linearity.

Author Contributions: Conceptualization, Y.L.; Data curation, Y.L.; Formal analysis, Y.L. and W.W.; Investigation, Y.L.; Methodology, Y.L.; Project administration, Y.H. and M.S.; Resources, Y.L.; Software, Y.L. and M.H.N.; Supervision, Y.H., M.S. and G.F.P.; Validation, Y.L.; Visualization, Y.L.; Writingoriginal draft, Y.L.; Writing—review and editing, Y.L., Y.H., M.H.N., F.J., W.W., J.R., Y.Y. and M.S. All authors have read and agreed to the published version of the manuscript.

Funding: This research was funded by China Scholarship Council, grant number 202004910734.

Conflicts of Interest: The authors declare no conflict of interest.

\section{References}

1. Cao, T.; Liu, Y.; Chen, W.; Yang, C.; Zhou, J. Analytical Design Solution for Optimal Matching of Hybrid Continuous Mode Power Amplifiers Suitable for a High-Efficiency Envelope Tracking Operation. Electronics 2019, 8, 621. [CrossRef]

2. Larsson, E.G.; Edfors, O.; Tufvesson, F.; Marzetta, T.L. Massive MIMO for next generation wireless systems. IEEE Commun. Mag. 2014, 52, 186-195. [CrossRef]

3. Inmarsat Massive MIMO for Next Generation Wireless Systems. Available online: https://arxiv.org/pdf/1304.6690.pdf (accessed on 23 January 2014).

4. Araniti, G.; Bisio, I.; De Sanctis, M.; Orsino, A.; Cosmas, J. Multimedia content delivery for emerging 5G-satellite networks. IEEE Trans. Broadcast. 2016, 62, 10-23. [CrossRef]

5. Lazzarin, G.; Pupolin, S.; Sarti, A. Nonlinearity compensation in digital radio systems. IEEE Trans. Commun. 1994, 42, 988-999. [CrossRef]

6. Mousavi, P.; Fakharzadeh, M.; Safavi-Naeini, S. 1K element antenna system for mobile direct broadcasting satellite reception. IEEE Trans. Broadcast. 2010, 56, 340-349. [CrossRef]

7. Habu, S.; Li, C.; Yamao, Y. Spectrum-folding scalar-feedback architecture for wideband DPD with simple feedback circuit. In Proceedings of the 47th European Microwave Conference (EuMC), Nuremberg, Germany, 10-12 October 2017; pp. $1054-1057$. 
8. Qiao, W.; Li, G.; Zhang, Y.; Li, H.; Liu, F. A band-limited digital pre-distortion method for hybrid MIMO transmitters. In Proceedings of the International Conference on Microwave and Millimeter Wave Technology (ICMMT), Guangzhou, China, 19-22 May 2019; pp. 1-3.

9. Ibarra, M.; Panduro, M.A.; Andrade, A.G. Differential evolution multi-objective for optimization of isoflux antenna arrays. IETE Tech. Rev. 2016, 33, 1-10. [CrossRef]

10. Khan, B.; Tervo, N.; Pärssinen, A.; Juntti, M. Average linearization of phased array transmitters under random amplitude and phase variations. In Proceedings of the 16th International Symposium on Wireless Communication Systems (ISWCS), Oulu, Finland, 27-30 August 2019; pp. 553-557.

11. Hammi, O.; Ghannouchi, F.M. Power alignment of digital predistorters for power amplifiers linearity optimization. IEEE Trans. Broadcast. 2009, 55, 109-114. [CrossRef]

12. Liu, X.; Zhang, Q.; Chen, W.; Feng, H.; Chen, L.; Ghannouchi, F.M.; Feng, Z. Beam-oriented digital pre-distortion for 5G massive MIMO hybrid beamforming transmitters. IEEE Trans. Microw. Theory Tech. 2018, 66, 3419-3432. [CrossRef]

13. Chen, W.; Lv, G.; Liu, X.; Feng, Z. Energy-efficient doherty power amplifier mmic and beamforming-oriented digital pre-distortion for 5G massive MIMO application. In Proceedings of the 2017 IEEE Asia Pacific Microwave Conference (APMC), Kuala Lumpur, Malaysia, 13-16 November 2017; pp. 391-394.

14. Tehrani, A.S.; Eriksson, T.; Fager, C. Modeling of long term memory effects in RF power amplifiers with dynamic parameters. In Proceedings of the IEEE/MTT-S International Microwave Symposium Digest, Montreal, QC, Canada, 17-22 June 2012; pp. 1-3.

15. Afsardoost, S.; Eriksson, T.; Fager, C. Digital pre-distortion using a vector-switched model. IEEE Trans. Microw. Theory Tech. 2012, 60, 1166-1174. [CrossRef]

16. Guo, Y.; Yu, C.; Zhu, A. Power adaptive digital pre-distortion for wideband RF power amplifiers with dynamic power transmission. IEEE Trans. Microw. Theory Tech. 2015, 63, 3595-3607. [CrossRef]

17. Chaplin, A.F. Analysis and synthesis of antenna arrays. NASA STI/Recon Tech. Rep. A 1987,88, 48703.

18. Niroo-Jazi, M.; Al-Saedi, H.; Gigoyan, S.; Safavi-Naeini, S. Design and analysis of reconfigurable reflectarray antennas for Ka-band satellite communications on the move. In Proceedings of the 17th International Symposium on Antenna Technology and Applied Electromagnetics (ANTEM), Montreal, QC, Canada, 10-13 July 2016; pp. 1-2.

19. Panduro, M.A.; Reyna, A.; Camacho, J. Design of scannable linear arrays with amplitude and phase optimisation for maximum side lobe level reduction. Int. J. Electron. 2009, 94, 323-329. [CrossRef]

20. Tervo, N.; Aikio, J.; Tuovinen, T.; Rahkonen, T.; Parssinen, A. Digital pre-distortion of amplitude varying phased array utilising over-the-air combining. In Proceedings of the IEEE MTT-S International Microwave Symposium (IMS), Honolulu, HI, USA, 4-9 June 2017; pp. 1165-1168.

21. Tervo, N.; Leinonen, M.E.; Aikio, J.; Rahkonen, T.; Parssinen, A. Analyzing the effects of PA variations on the performance of phased array digital pre-distortion. In Proceedings of the IEEE 29th Annual International Symposium on Personal, Indoor and Mobile Radio Communications (PIMRC), Bologna, Italy, 9-12 September 2018; pp. 215-219.

22. Moradi, A.; Mohajeri, F. Side lobe level reduction and gain enhancement of a pyramidal horn antenna in the presence of metasurfaces. IET Microw. Antennas Propag. 2018, 12, 295-301. [CrossRef]

23. Yu, C.; Guan, L.; Zhu, E.; Zhu, A. Band-limited volterra series-based dig ital pre-distortion for wideband RF power amplifiers. IEEE Trans. Microw. Theory Tech. 2012, 60, 4198-4208. [CrossRef]

24. Choi, S.; Jeong, E. Digital pre-distortion based on combined feedback in MIMO transmitters. IEEE Commun. Lett. 2012, 16, 1572-1575. [CrossRef]

25. Lee, S.; Kim, M.; Sirl, Y.; Jeong, E.; Hong, S.; Kim, S.; Lee, Y.H. Digital pre-distortion for power amplifiers in hybrid MIMO systems with antenna subarrays. In Proceedings of the IEEE 81st Vehicular Technology Conference (VTC Spring), Glasgow, UK, 11-14 May 2015; pp. 1-5.

26. Liu, L.; Chen, W.; Ma, L.; Sun, H. Single-PA-feedback digital pre-distortion for beamforming MIMO transmitter. In Proceedings of the IEEE International Conference on Microwave and Millimeter Wave Technology (ICMMT), Beijing, China, 5-8 June 2016; pp. 573-575.

27. Abdelaziz, M.; Anttila, L.; Brihuega, A.; Tufvesson, F.; Valkama, M. Digital pre-distortion for hybrid MIMO transmitters. IEEE J. Sel. Top. Signal Process 2018, 12, 445-454 [CrossRef]

28. Luo, Q.; Zhu, X.; Yu, C.; Hong, W. Single-receiver over-the-air digital pre-distortion for massive MIMO transmitters with antenna crosstalk. IEEE Trans. Microw. Theory Tech. 2020, 68, 301-315. [CrossRef]

29. Li, Y.; Huang, Y.; Shen, M. Modeling and linearization of active phased arrays with non-uniform power distribution. In Proceedings of the 27th Telecommunications Forum (TELFOR), Belgrade, Serbia, 26-27 November 2019; pp. 1-4.

30. Alicano, C.R.; Covarrubias, D.H.; Brizuela, C.A.; Panduro, M.A. Differential evolution algorithm applied to sidelobe level reduction on a planar array. Int. J. Electron. Commun. 2007, 61, 286-290. [CrossRef]

31. Reyna, A.; Panduro, M.A.; Covarrubias, D.H.; Mendez, A. Design of steerable concentric rings array for low side lobe level. Sci. Iran. 2012, 19, 727-732. [CrossRef]

32. Cucini, A.; Albani, M.; Maci, S. Truncated floquet wave full-wave analysis of large phased arrays of open-ended waveguides with a nonuniform amplitude excitation. IEEE Trans. Antennas Propag. 2003, 51, 1386-1394. [CrossRef]

33. Chen, F.; Chen, J.; Chu, Q.; Lancaster, M.J. X-band waveguide filtering antenna array with nonuniform feed structure. IEEE Trans. Microw. Theory Tech. 2017, 65, 4843-4850. [CrossRef] 
34. Buttazzoni, G.; Babich, F.; Vatta, F.; Comisso, M. Geometrical synthesis of sparse antenna arrays using compressive sensing for 5G IoT applications. Sensors 2020, 20, 350. [CrossRef]

35. Panduro, M.A.; Mendez, A.L.; Dominguez, R.; Romero, G. Design of non-uniform circular antenna arrays for side lobe reduction using the method of genetic algorithms. Int. J. Electron. Commun. 2006, 60, 713-717. [CrossRef]

36. Zhu, A.; Pedro, J.C.; Brazil, T.J. Dynamic deviation reduction-based volterra behavioral modeling of RF power amplifiers. IEEE Trans. Microw. Theory Tech. 2006, 54, 4323-4332. [CrossRef]

37. Matsubara, H.; Ishihara, K.; Miyadai, N.; Nojima, T. Hybrid pre-distortion to compensate third- and fifth-order intermodulation of a $2 \mathrm{GHz}$ power amplifier using cuber pre-distortion and second harmonics injection. IET Microw. Antennas Propag. 2008, 2, 813-822. [CrossRef]

38. Soleiman, E.; Germain Pham, D.; Jabbour, C.; Desgreys, P.; Kamarei, M. Memory aware physically enhanced polynomial model for PAs. IET Microw. Antennas Propag. 2019, 13, 833-841. [CrossRef]

39. CGH40006, 6 W, GaN HEMT by Cree for General Purpose (CGH40006p) (Wireless Devices) Transistor. Available online: https:/ /www.mouser.com/d/s/2/90/gh40006p-876367.pdf (accessed on 14 May 2015)

40. Nunes, L.C.; Cabral, P.M.; Pedro, J.C. AM/AM and AM/PM distortion generation mechanisms in Si LDMOS and GaN HEMT based RF power amplifiers. IEEE Trans. Microw. Theory Tech. 2014, 62, 799-809. [CrossRef]

41. Std-T, Arib Evolved Universal Terrestrial Radio Access (E-UTRA); User Equipment (UE) Radio Transmission and Reception (Release 10). Available online: https://www.arib.or.jp/english/html/overview/doc/STD-T104v3_10/4_Cross_Reference/ 3GPP_SPEC_Cross_ReferenceT104V310_Rel10.pdf (accessed on 30 September 2015).

42. Gilmore, R. Practical rf circuit design for modern wireless systems v.ii. IEEE Electr. Insul. Mag. 2004, $20,54$.

43. Gilmore, R. Satellite Communications Antenna Concepts and Engineering; Springer: New York, NY, USA, 2013.

44. Reyna, A.; Panduro, M.A.; del Rio-Bocio, C.; Méndez, A.L. Design of different planar geometries of antenna arrays for isoflux radiation in GEO satellites. Telecommun. Syst. 2017, 65, 269-279 [CrossRef] 